Du texte à la scène : langages du théâtre

\title{
Table ronde sur la traduction théâtrale
}

Jean Fuzier

\section{(2) OpenEdition \\ Journals}

Édition électronique

URL : http://journals.openedition.org/shakespeare/488

DOI : $10.4000 /$ shakespeare.488

ISSN : 2271-6424

Éditeur

Société Française Shakespeare

Édition imprimée

Date de publication : 1 novembre 1983

Pagination : 274-277

Référence électronique

Jean Fuzier, "Table ronde sur la traduction théâtrale », Actes des congrès de la Société française

Shakespeare [En ligne], 4 | 1983, mis en ligne le 01 janvier 2007, consulté le 04 mai 2019. URL : http:// journals.openedition.org/shakespeare/488; DOI : 10.4000/shakespeare.488 
SOCIETE FRANÇAISE SHAKESPEARE

Actes du Congrès 1982

\section{DU TEXTE A LA SCENE : Langages du Théâtre}

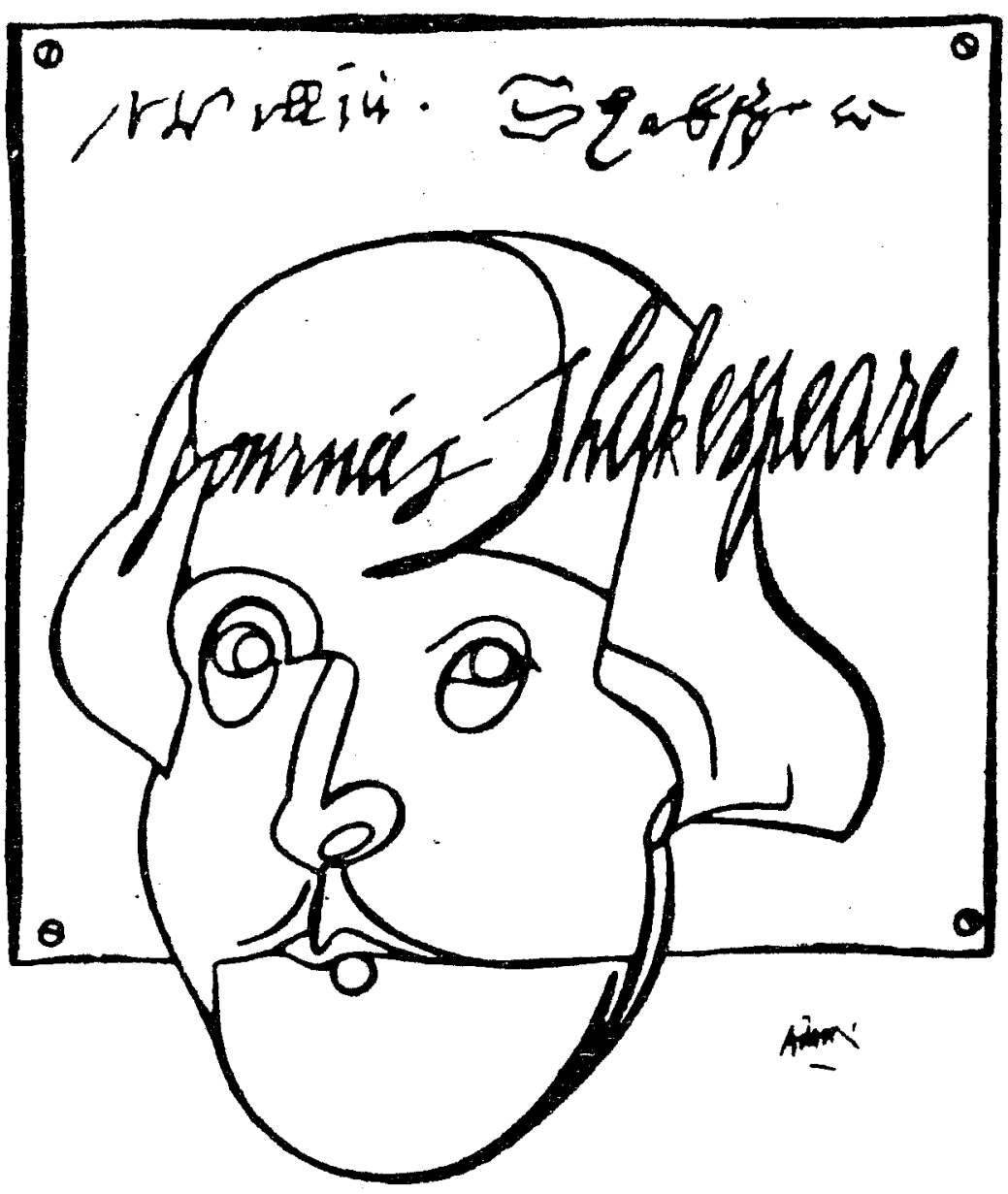

DiRecteur de la publication M.T. Jones - Davies

Publié avec le concours du Centre National de la Recherche Scientifique

JEAN TOUZOT Libraire - Editeur

38 , rue Saint-Sulpice 75278 PARIS CEDEX 061983 
TABLE RONDE (dirigée par Jean Fuzier)

\section{RESUME DES INTERVENTIONS SUR LA TRADUCTION THEATRALE}

Lundi 11 octobre 1982 après-midi.

Jeen-Michel DEPART : Traduit-on différemment selon que l'on destine une traduction à la lecture ou à la scène? En droit cette distinction n'a pas lieu d'être. Une traduction impraticable sur une scène, régie par une poétique de l'écrit, méconnaît une dimension essentielle du texte shakespearien, tout entier tendu vers la représentation. Le texte de Shakespeare, écrit par un acteur pour des acteurs, est un texte de théâtre où l'ordre des mots, les rythmes, les images sont porteurs de gestes, où les propriétés sensibles du verbe sont un instrument de jeu pour le comédien. Prendre en compte l'exigence du jeu, ce n'est donc pas porter atteinte au flamboiement imaginatif et à la texture poétique, c'est au contraire tenter de préserver les particularités de l'écriture, car l'abondance des métaphores a pour fonction théâtrale d'accroître la tension de l'élocution et l'énergie de la diction.

La difficulté majeure est dans la raideur prosodique et les strictes exigences grammaticales et syntaxiques du français. Celui-ci s'accommode mal du jaillissement métaphorique et du resserrement elliptique de la pensée de Shakespeare. Le combat principal est contre la tyrannie d'un cartésianisme linguistique et mental qui conduit à expliciter, édulcorer, enjoliver, «grammaticaliser», réduire, pour obéir à des critères que l'on croit consubstantiels au génie de la langue français : l'aisance, la clarté, la logique. Résister à l'explication, à la normalisation sur les codes syntaxiques et lexicaux du français, c'est tenter cic :'culer les limites, préserver l'étrangeté des propositions, la violence d'une métaphore ou le rayonnement d'un mot. Quitte à faire un faux sens, à tordre l'usage habituel ou l'ordre normal des mots. Naturellement, bien d'autres 
exigences entrent en jeu que le traducteur ne peut manquer de hiérarchiser et qu'il ne prétend pas toutes satisfaire.

Jean Fuzier : L'expérience théâtrale montre qu'on est obligé de modifier à la scène, au cours des répétitions, le texte de la traduction (par exemple le Roméo et Juliette de Cacoyannis joué à Paris en 1968, pour lequel il a fallu adapter la traduction de Copeau).

D'autre part les traductions, même quand elles sont les meilleures de leur temps, comme celles de Pitoëff, sont rapidement dépassées.

Les coupures sont un problème de théâtre, non de traduction; on coupait déjà les textes de la moitié de la longueur au temps de Shakespeare, pour le théâtre; quand on traduit pour la scène, on traduit intégralement et on coupe ensuite. Il y a aussi les modifications dues au goût du public (le mouchoir de Vigny).

La traduction des Sonnets est une autre question; j'ai dû traduire par des formes et des mètres à peu près équivalents, alors que pour le théatre, il faut une prosodie plus souple. Quand il y a dans une pièce un passage rimé et versifié à des fins particulières - tirade parodique, sonnet, poème, chanson - il est hors de question de traduire autrement que par une forme métrique régulière; et quand il y a à la fois vers et prose, il faut qu'à l'oreille ce qui est vers se distingue de ce qui est prose.

Michel Grivelet : La traduction pour la lecture et la traduction pour la scène sont deux choses différentes.

Copeau, pour son Soir des Rois, a écrit dans Les Mémoires du Vieux Colombier qu'à mesure des répétitions, ils ont modifié le texte, le traducteur n'ayant pas eu l'occasion d'éprouver la traduction du point de vue sonore sur l'espace scénique; la traduction pour le théâtre est immédiatement subordonnée à la conception de la mise en scène.

Il est inconcevable qu'on traduise le sens sans traduire 
d'abord les images; le sens réside dans les images. Dans Henry $I V$, dans la scène de la taverne, si on traduit «What time of day is it, lad?» par «quelle heure est-il ?» on s'apercevra avant la fin de la scène que cela ne convient pas : il s'agit de l'heure du jour, qui n'intéresse pas Falstaff, chevalier de la nuit, et on ne doit pas sauter cette nuance. Il faut suivre l'ordre des mots, la couleur dans la phrase de Shakespeare, le concret des mots, le concret des images, le «word play», le «punning». Le mot anglais bifurque dans tous les sens. Il faut traduire au profit de la langue traduite, et non au profit de la langue de traduction. Il faut se laisser envahir par le texte de Shakespeare, s'exposer à ce «flash»; c'est une question d'expérience humaine plutôt que d'expérience littéraire qui engage toute l'âme et la conscience, quitte à procéder à des ajustements à la lumière des traductions antérieures. Il faut aussi laisser transparaître à la scène la différence entre la première scène de Henry $I V$ qui est en vers et la deuxième qui est en prose; c'est une des raisons pour lesquelles il faut distinguer la traduction pour la lecture et la traduction pour la scène : sur la page imprimée, on a le secours de la typographie qui fait apparaître les vers : il faut un autre moyen de la faire entendre sur scène.

Nato Israel : Faut-il traduire ou adapter les textes étrangers (exemple Shakespeare)? Doit-on retracer ou abolir la distance culturelle entre les époques, les idiomes et les peuples?

Traductions savantes : visent à rendre l'intégralité de l'oeuvre (ex. Hugo, traductions juxta-linéaires) posent le problème de la nature et des limites de l'exactitude (non coincidence des systèmes linguistiques, connotation, polysémie, interférence du traducteur, irréductibilité du message au seul contenu sémantique ...). Une traduction trop exacte peut entraîner des tensions voire des ruptures, et constitue un instrument d'élucidation plutôt qu'un objet littéraire et dramatique.

Adaptations : plus autonomes. Recherchent souplesse 
et naturel et se préoccupent davantage de la langue-cible, du code théâtral, de la réception, mais sacrifient volontiers le détail à l'ensemble, la précision à l'effet. Appropriation parfois excessive quand l'auteur donne libre cours à sa spontanéité ou fait trop de concessions au goût du jour (ex. Ducis, Vigny).

Opposition entre l'unicité, la pérennité du textesource et le nombre, la diversité et le vieillissement rapide des équivalents proposés qui sont le reflet d'un certain état de la langue et de la sensibilité. Traduire c'est choisir c'est-à-dire limiter le champ des possibles, offrir une approximation. Toute traduction recèle une part d'adaptation. De même toute adaptation doit être en même temps une traduction sous peine de devenir un objet autonome se substituant à l'original. D'où la nécessité pour la réussite de l'opération traduisante de trouver un équilibre entre littéralité et transposition, décodage et recréation, qui tienne compte le plus possible de la spécificité dramatique de l'œuvre et de ses divers niveaux de signification (ex. Gide, Bonnefoy).

Henri Suhamy : Il est regrettable de ne pas disposer d'une traduction contemporaine, l'équivalent par exemple de ce que Florio a fait pour Montaigne dans l'autre sens, car la souplesse et la prolifération lexicale du français de la Renaissance auraient peut-être permis de restituer quelque chose de la liberté et de l'invention linguistique qui caractérisent le discours shakespearien. Car il ne faut pas se tromper : on dit par une périphrase de routine que l'anglais est la langue de Shakespeare, mais c'est partiellement faux car l'anglais de Shakespeare est un idiolecte qui n'appartient qu'à lui, matériau d'une poésie infinie, obscure, enchevêtrée, polysémique. Les traductions qui ne font qu'expliquer et simplifier, et qui vont parfois jusqu'à décoder les métaphores, annulent la poésie sans être même assurées d'éviter le contresens. Ne traduire qu'un sens parmi tous ceux qui sont imbriqués les uns dans les autres équivaut souvent à commettre une erreur. Il ne faut pas croire que les traducteurs médiocres man- 
quent seulement de virtuosité et d'inspiration. La difficulté intrinsèque du texte n'est pas toujours prise en considération. Il y a encore des traducteurs de Shakespeare qui ne font que traduire ce qu'ils comprennent, c'est-à-dire pas grand' chose. Shakespeare en français, c'est le plus souvent le festival du prosaïsme et du contresens harmonieusement associés.

Je rêve d'une traduction essentiellement poétique, ou plutôt d'une traduction qui serait un poème, écrit dans une langue qui ne serait pas vraiment du français, mais $d u$ Shakespeare transposé en français, qui emplirait le théâtre comme un nuage opaque et iridescent.

Les tentatives de traductions en vers méritent d'être encouragées, mais l'alexandrin classique tend un peut trop à s'organiser en deux hémistiches de six syllabes, plutôt qu'en une coulée de douze syllabes, ce qui produit en fin de compte un mètre un peu trop court.

Dans tous les cas la traduction des passages écrits en vers doit être juxta-linéaire, et doit s'efforcer de rendre le style, le mouvement de la pensée, en essayant de se calquer sur la syntaxe shakespearienne dans ce qu'elle a d'insolite et de tourmenté. La traduction de Virgile par Klossowski, celle de Proust par Scott Moncrieff, pourraient servir de modèle, ou du moins de stimulants. Il n'y a pas d'incompatibilité entre la. poésie et l'exactitude : on atteint chacune à travers l'autre, du moment que la notion d'exactitude inclut le souci de la forme.

Jean-Pierre Villquin: à propos de sa traduction de Roméo et Juliette pour la scène.

Les traductions pour le théâtre viellissent vite et mal : celles qui survivent sont irrémédiablement désuètes, trop méticuleuses ou trop académiques et ne répondent pas aux cxigences de la scène contemporaine. Si on ne veut pas que le texte de Shakespeare soit un simple prétexte, chaque nouvelle mise en scène demande une nouvelle traduction.

Il faut un texte que le comédien puisse «mettre en 
bouche», car pour lui c'est une mécanique sur laquelle se règle la respiration, avec ses jeux d'allitérations et d'assonances, avec ses accents et ses pauses. Il faut aussi un texte qui respecte le lyrisme, la parodie, la violence et les jeux de mots. Il n'était pas question d'encaquer systématiquement le vers shakespearien dans les limites étriquées d'un alexandrin de pacotille, pourtant, chaque fois qu'il est venu naturellement, je me suis attaché à cette discipline métrique pour la densité et le rythme qu'impose la prononciation exacte des syllabes. C'est la texture sonore des mots que j'ai voulu rendre d'abord, car il y a une sorte de partition musicale non écrite qui accompagne le foisonnement d'images et la richesse des mots. C'est en effet dans les mots, plus que dans le décor ou le jeu des acteurs que réside la force de l'illusion.

Quatre siècles nous séparent de Shakespeare, le langage, ses images et ses conventions ne sont plus tout à fait les nôtres, un décodage serait nécessaire mais la scène ne supporte pas les explications, le public doit comprendre d'emblée. La solution relève moins du domaine de la sémantique que de celui de la sensation, c'est pourquoi la complexité, l'éclat et l'exubérance des métaphores, la densité et la multiplicité des sens ne peuvent être rendus que par le choix et la mise en valeur de mots «relais», ainsi un processus analogique, par accumulation de mots qui se font écho, permet la compréhension.

La poésie au théâtre est ancrée au monde du concret et au monde de l'action, elle donne ainsi aux «choses qui ne sont pas des choses» une nouvelle dimension. Les jeux de mots qui fusent comme des balles échangées ne peuvent trouver un support efficace que dans l'habileté, la virtuosité du geste et du mouvement; l'art plastique vient conforter, expliquer les subtilités du langage car le théâtre n'est pas fait que de mots. Pour garder vivant le théâtre de Shakespeare, le mieux est de se laisser porter par le flot si, comme je le crois, le rôle du traducteur est d'abord de se faire oublier. 